'|||||||||||||||||||||||||||||||||||||||||||||||||||||||||||||

\section{Der Apfel der Weisen}

Liebe Leserin, lieber Leser,

die oft als ach so rückständig gescholtene deutsche Autoindustrie bringt sich in Stellung, indem sie das Glück der Partnerschaft entdeckt. Aktuelles Beispiel: Bosch bandelt mit Microsoft an und will auf dessen Azure-Cloudumgebung basierend eine Softwareplattform für die „nahtlose Vernetzung von Fahrzeugen“ schaffen. Ziel der Kooperation sei es, die Fahrzeugsoftware stetig weiterzuentwickeln und diese dann auf den Fahrzeugrechnern zu aktualisieren. Damit wird der Zustand, dass Fahrzeuge ab dem SoP einen mehr oder minder eingefrorenen Zustand von Fähigkeiten und Funktionen aufweisen, komplett aufgehoben. Sie sind dann eher, die Phrase sei erlaubt, bis zum Ende des Lebenszyklus im Zustand „panta rhei“. Der ewige Fluss von neuen Softwareständen, Einstellungen und Fähigkeiten von Fahrzeugassistenz- bis hin zu sicherheitsgerichteten Funktionen wie Bremsparametern oder Dämpfereigenschaften bedeutet eine fast völlige Loslösung von der Definition einer Homologation.

Neben Microsoft ist auch Amazon Web Services (AWS) bei den OEMs derzeit sehr gefragt, auf anderen Ebenen Dienstleister wie T-Systems oder NTT. Die Anwendungsbereiche dieser Data-MiningPartnerschaften reichen von der Entwicklung bis in die Fertigung oder die Umsetzung des automatisierten Fahrens hinein, aber natürlich sind auch die Themen OTA und Cybersecurity relevant. Vor diesem Hintergrund kann man eine tiefgreifende Wandlung des Fahrzeugs und auch der Aufstellung der Automobilhersteller und -zulieferer feststellen. Neben der Elektrifizierung der Fahrzeuge findet hier die vierte industrielle Revolution in der Fahrzeugtechnik statt: „The future is electric“ ist ein viel genutzter Spruch, aber zugleich ist die Zukunft - nicht nur in Bezug auf die Fahrzeugtechnik vor allem elektronisch beziehungsweise datengetrieben. Für diese Entwicklung wird neben einem entsprechenden Backbone beziehungsweise einer Middleware im Fahrzeug auch das im Englischen gern als Eco System bezeichnete Szenario für das Handling und die Nutzung der riesigen Datenmengen benötigt. Zieht man Parallelen zu Rechner-erzeugten Bitcoins und deren enormem Energiebedarf, kann einem angst und bange in Sachen Ressourcenverbrauch der schönen neuen Welt hochautomatisierter, elektrisch angetriebener Fahrzeuge werden. WLTP allein mag da kein Maßstab mehr für den Benzinverbrauch auf 100 km sein: Der momentan als Stein der Weisen empfundene Trend zu sauberen Elektrofahrzeugen kann durch die Ergänzung mit HAD-Systemen genauso gut zu einem Sündenfall in Sachen Energiebedarf werden. Und das noch bevor Apple sein von vielen herbeibeschworenes iCar auf den Markt bringt.

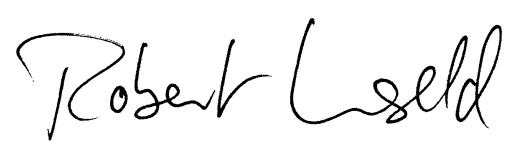

Robert Unseld

Verantwortlicher Redakteur

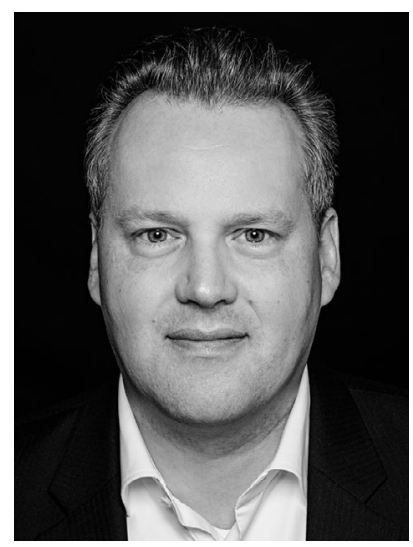

